INPLASY

PROTOCOL

To cite: Yu et al. The efficacy and safety of Health Qigong for anti-aging: Protocol for a systematic review and metaanalysis. Inplasy protocol 202090017. doi:

10.37766/inplasy2020.9.0017

Received: 04 September 2020

Published: 04 September 2020

Corresponding author:

Peng Yu

yp1993216@163.com

Author Affiliation:

Chengdu University Of

Traditional Chinese Medicine

Support: No support.

Review Stage at time of this submission: Preliminary searches.

Conflicts of interest:

The author have no conflicts of interest.

\section{The efficacy and safety of Health Qigong for anti-aging: Protocol for a systematic review and meta-analysis}

Yu, P1; Li, W2; Li, H³; Ouyang, S4; Cai, H5; Wu, J6; Tang, C7; Huang, $Q^{8}$.

Review question / Objective: Is the Health Qigong effective and safe for anti-aging? Can Health Qigong regulate immune function and enhance the antioxidant capacity? This review is intended to find the answers.

Condition being studied: Aging is a natural process which is generally along with a decline in the physiological function of the body, as a result of the susceptibility to age-associated diseases will be increased.For example, cardiovascular disease, dementia, osteoporosis, diabetes, hypertension, and stroke are age-induced diseases.Aging brings several diseases together will produce destructive effects on human society and breakdowns the entire health care system and economy.

INPLASY registration number: This protocol was registered with the International Platform of Registered Systematic Review and Meta-Analysis Protocols (INPLASY) on 04 September 2020 and was last updated on 04 September 2020 (registration number INPLASY202090017).

\section{INTRODUCTION}

Review question / Objective: Is the Health Qigong effective and safe for anti-aging? Can Health Qigong regulate immune function and enhance the antioxidant capacity? This review is intended to find the answers.

Rationale: The oxidative stress and free radical accumulation theories stand out the most in the multiple theories related with aging. Additionally oxidative stress, aging 
is also intimately related with issuing in structural and functional flaws in the immune system. Health Qigong, as a traditional Chinese exercise, has a remarkable effect on improving immune function and he antioxidant capacity of the body.

Condition being studied: Aging is a natural process which is generally along with a decline in the physiological function of the body, as a result of the susceptibility to age-associated diseases will be increased. For example, cardiovascular disease, dementia, osteoporosis, diabetes, hypertension, and stroke are age-induced diseases.Aging brings several diseases together will produce destructive effects on human society and breakdowns the entire health care system and economy.

\section{METHODS}

Search strategy: We will search electronic databases, such as PubMed, EMBASE, Web of Science, the Cochrane Library, CNKI, Wangfang databases, the Chinese Biomedical Literature Database (CBM), the Chinese Science and Technology Periodical Database (VIP) and the Chinese Cochrane Centre's Clinical Trial Registry Platform. The relevant literature will be selected without the language restriction, using keywords related to Health Qigong and anti-aging.

Participant or population: Subjects aged 45 to 75 years, who do not exercising daily and have no major disease will be selected. There will be no restriction on gender, race, or nation.

Intervention: The intervention group uses Health Qigong as a separate exercise, and the specific operation methods were as follows. Health Qigong include Baduanjin, Wuqinxi , Yijinjing, Liuzijue and TaiChi.

Comparator: The control group has the following forms: keep previous exercise habit, restrict any exercise etc.

Study designs to be included: RCTs will be included in our study. If the number of RCT literatures included is less than 6 , CCSs will also be included.

Eligibility criteria: Only the RCTs of Health Qigong for anti-aging will be included in this study, without placing the constraint on publication status and writing language. Studies without sufficient information about the randomized method or process, the animal mechanism studies, qualitative studies, uncontrolled trials and reviews and case reports will be excluded.

Information sources: The following 7 English databases ( PubMed, Excerpta Medica Database, MEDLINE, Web of Science, Cochrane Library, SpringerLink, and WHO International Clinical Trials Registry Platform) and 4 Chinese databases ( namely the China National Knowledge Infrastructure Database, the Wanfang Database, the Chinese Scientific Journal Database, and the Chinese BioMedical Literature Database) will be investigated from their inception to August, 2020. The search strategy for selecting the fields of topic, title, or abstract was unique referring to the characteristics of databases. The complete PubMed search strategy is in Table 1 and will be modified and used in other electronic databases.

Main outcome(s): The main outcomes include the antioxidant capacity and the immune function. The antioxidant capacity are total antioxidant capacity (T$A O C$ ), superoxide dismutase (SOD) and Malondialdehyde (MDA). The immune function index are TNF-a counts, IFN- $\gamma$ counts, IL-2 counts, IL- 6 counts and the level of $T$ cell subsets (CD3+, CD4+, CD8+, CD4+/CD8+).

Additional outcome(s): The Secondary outcomes are cardio-cerebrovascular function and cognitive competence.

Data management: Two researchers will independently work for data extraction, they will collect the undermentioned data: (1) basic characteristics, including the first author, publication year, sample size, trial location, age, geographic population, health status, duration and follow-up, 
frequency, intensity, Health Qigong style, control intervention. (2)primary outcomes and secondary outcomes as the information showed before will also be included, including the antioxidant capacity, the immune function and cardiocerebrovascular function. This process will be accomplished by 2 researchers, and the results will be double-verified by an another researcher. If the data is missing or unclear, the corresponding author will be contacted.

Quality assessment / Risk of bias analysis: We will according to the Cochrane Handbook of Systematic Reviews of Interventions, and the risk of bias(ROB) of the included studies will be assessed by two researchers independently using the Cochrane risk of bias assessment tool. The domains pertinent to ROB including random sequence generation, allocation concealment, blinding of participants and therapist, blinding of outcome assessment, incomplete outcome data, selective reporting, and other bias. The ROB for each domain will be rated as"low risk," "high risk," or "unclear risk."

Strategy of data synthesis: The metaanalysis will be performed by using the Review Manager V.5.3 (RevMan 5.3)software.We will describe the effect size with the risk Ration(RR) for dichotomous data, and the mean deference(MD) for continuous data $.95 \%$ of the confidence interval $(\mathrm{Cl})$ will be used as an effective size for the combined analysis.

Subgroup analysis: If we have a sufficient number of RCTs for inclusion in the review, we will carry out the subgroups when the heterogeneity is high. Subgroup analysis will be carried out based on the type of control interventions, type of Health Qigong, exercise frequency and duration.

Sensibility analysis: We will conduct the sensitivity analysis to assess the robustness of bias on results and the sources of heterogeneity. We will delete the data of certain study one by one by comparing the recombined date with original date, and then focus on the changes merged size effects and heterogeneity.

Language: Chinese and english.

Country(ies) involved: China.

Keywords: Health Qigong, aging, antiaging, systematic review.

Dissemination plans: The results of our research will be published at a peer reviewed journal.

Contributions of each author:

Author 1 - Peng Yu - The author designed the experimentd and drafted the manuscript.

Author 2 - Weihong Li - The author provided statistical expertise and supervised the experiment.

Author 3 - Han Li - The author accomplished data collection and analysis.

Author 4 - Shuang Ouyang - The author accomplished data collection and analysis.

Author 5 - Haiyang Cai - The author accomplished data extraction risk of bias assessment.

Author 6 - Jing Wu - The author accomplished data extraction risk of bias assessment.

Author 7 - Chaohui Tang - Describe contributions of each author.

Author 8 - Qingsong Huang - The author read, provided feedback. 